\title{
LA VINDICACIÓN DE LOS DERECHOS DE LA MUJER ANTES DE MARY WOLLSTONECRAFT ${ }^{1}$
}

\author{
María Jesús Lorenzo Modia \\ Universidade de A Coruña
}

\begin{abstract}
This paper explores the intellectual and cultural background that preceded the publication of $A$ Vindication of the Rights of Woman by Mary Wollstonecraft in 1792. This writer cannot be viewed as an exception in the history of European women. On the contrary, she is a link -albeit outstanding- with the eighteenth-century feminist or proto-feminist thought prevalent in Europe that antedated and transcended both the English and the French revolutions, and that still permeates the culture of the old continent.
\end{abstract}

\section{INTRODUCCIÓN}

El título de este trabajo intenta resumir la idea que trataré de desarrollar a continuación, esto es, que la larga marcha por el reconocimiento de los derechos de las mujeres es en muchas ocasiones desconocida, porque la cultura femenina se ha transmitido primordialmente de manera oral y está condenada a ser una cultura ágrafa, que no se ha valorado ni difundido suficientemente en el patriarcado. A pesar de que lo que conocemos hoy en día son los textos que sobre este tema vieron la luz, y que incluso pueden haberse convertido en emblemáticos, esta lucha no comienza con la publicación de los mismos, sino que es justamente al contrario, la lucha por la equiparación de los derechos de los sexos proviene del intento de subversión del statu quo, y que sólo en último término se transmite mediante publicaciones, de las que sus autoras o autores son únicamente los mediums por los que se transmiten ideas subyacentes. A veces, estos intentos no son suficientemente ponderados en su tiempo y es precisa la perspectiva histórica para su puesta en valor. Si el reconocimiento intelectual a las mujeres, y por ende, la publicación de textos de autoría femenina es en sí misma casi una excepción en pleno siglo XXI; no es, pues, de extrañar que este reconocimiento fuese aun más dificultoso en épocas precedentes cuando la situación social, cultural y personal de las mujeres era mucho más dura y complicada. Como consecuencia de esta idea mi intención es proponer un recorrido por diferentes obras y autores que conformaron el contexto intelectual europeo anterior a la publicación por Mary Wollstonecraft de la Vindicación de los derechos de la mujer, en el Londres de 1792.

He elegido la figura de Mary Wollstonecraft como referencia, ya que se la considera la primera autora europea que publica un ensayo sistematizado sobre la situación de la mujer

\footnotetext{
${ }^{1}$ Este trabajo se ha realizado con las ayudas respectivas del Ministerio de Educación y Ciencia, a través del Proyecto de Investigación BFF2003-0065 "Literatura y violencia de género", y de la Universidade da Coruña, mediante el Proyecto de Investigación "Literatura de autoría femenina en lengua inglesa del siglo XVIII", que aquí se agradecen expresamente.
} 
y que propone explícitamente el reconocimiento de sus derechos: $A$ Vindication of the Rights of Woman, traducido al castellano como Vindicación de los derechos de la mujer, publicado por Cátedra y el Instituto de la Mujer en 1994, en una edición de Isabel Burdier. El pequeño cambio en el título, omitiendo el artículo indeterminado que en inglés antecede a vindicación, sirve -en mi opinión- como merecido homenaje a un texto considerado ahora en Europa como pionero, y al que se le concede la categoría de texto fundador $-\mathrm{y}$ al tiempo perfectamente actual- de la lucha por la igualdad.

La vida personal y profesional de Mary Wollstonecraft refleja con claridad su percepción de las dificultades de las mujeres en el mundo hostil en el que viven y la intención de modificar tanto la legislación vigente como la praxis vital para cambiarlo. Asimismo, su vida da testimonio del interés que ésta tenía por la educación de la mujer y por su acceso a la cultura desde la infancia y constata el deseo de la autora por la independencia profesional mediante la docencia y la profesión literaria. En este aspecto, su obra abarca aspectos tan variados como la edición, la traducción, la obra destinada a niños, la ficción y el ensayo.

Su obra más conocida es la Vindicación de los derechos de la mujer, que escribió tras la publicación en 1790 de la Vindicación de los derechos del hombre, y que refleja una crítica a la sociedad existente y a su discurso androcéntrico. El ensayo que vio la luz en 1792 presenta una propuesta de igualdad entre hombres y mujeres a través de la educación y de la reforma de la legislación.

\section{LA TRADICIÓN EN ESPAÑA}

El panorama ideológico e intelectual europeo en el siglo XVIII es en general conocido como la Ilustración. Sin embargo, con relación al 'problema de la mujer' no se plantean intelectualmente grandes cambios. En el contexto español, a pesar de que aún tenían vigencia y se citaban con frecuencia como modelos de conducta para la mujer textos como La instrucción de la mujer cristiana (1525) de Juan Luis Vives o La perfecta casada (1583) de Fray Luis de León, el siglo XVIII supone un gran avance, al menos en lo que respecta a una mayor preocupación por la situación de la mujer.

No debemos olvidar, por ejemplo, al Padre Feijóo, que ya en 1726 escribió su Discurso XVI del volumen primero del Teatro crítico denominado Defensa de la mujer (Feijóo 1997), con "un espíritu libre y emancipador" (Sau 1997: 11), ya que "la tónica general en el Discurso es la de que la mujer, en muchos casos, excede incluso en aptitudes o entendimiento al hombre" (Sau 1997: 9). Sin embargo, el propio Feijóo ha de empezar su discurso con una justificación del mismo, puesto que sabe que su posición no es unánimemente aceptada:

En grave empeño me pongo. No es ya sólo un vulgo ignorante con quien entro en la contienda: defender a todas las mujeres, viene a ser lo mismo que ofender a casi todos los hombres, pues raro es el que no se interesa por la procedencia de su sexo con desestimación del otro. A tanto se ha extendido la opinión común en vilipendio de las mujeres, que apenas admite en ellas cosa buena. En lo moral las llena de defectos, y en lo físico de imperfecciones. Pero donde más fuerza hace, es en la limitación de sus entendimientos. Por esta razón, después de defenderlas con alguna brevedad sobre otros capítulos, discurriré más largamente sobre su aptitud para todo género de ciencias y conocimientos sublimes (Feijóo 1997: 15). 
Asimismo, conviene destacar a dos autoras españolas contemporáneas de Wollstonecraft como Josefa Amar y Borbón e Inés Joyes y Blake. Amar y Borbón destaca en su Discurso sobre la educación física y moral de las mujeres la necesidad de la educación de las mujeres y sostiene, paradójicamente, que las tareas adecuadas para ellas son las del ámbito doméstico. (Palacios 2002: 56- 57). Por su parte, Inés Joyes y Blake escribió en 1798 una "Apología de las mujeres", donde argumenta que la mujer no es inferior al hombre por naturaleza, sino que es el contexto social y educativo el que la hace inferior (Pajares 2000: 188-189).

La obra Vindicación de los derechos de la mujer de Wollstonecraft no vio la luz como tal en España, sino que fue recensionada en una serie de cuatro artículos publicados en el Diario de Madrid por su coeditor Julián de Velasco a partir del 6 de septiembre de 1792. Como era habitual, la versión utilizada para la traducción fue una traducción francesa y no la original inglesa. Julián de Velasco realizó una crítica muy favorable de la obra, incluyendo algunos de los párrafos de la misma, principalmente aquellos fragmentos que mejor representaban el ideario central de la autora. Ahora bien, no duda en omitir toda referencia crítica de Wollstonecraft a la opresión ejercida en la sociedad por parte de la aristocracia, la monarquía y la Iglesia, y que sin duda podrían ocasionarle problemas con la censura inquisitorial y eclesiástica operante en aquellos momentos en España (Kitts 1994: 353- 56).

\section{LA TRADICIÓN EN FraNCIA}

Debido a nuestros desencuentros históricos con la denominada pérfida Albión, la tradición británica es probablemente menos conocida en España que la aportación de los intelectuales franceses que antecedieron a Mary Wollstonecraft en su idea de que la mujer fuese considerada en igualdad de derechos y deberes. Así se citan con frecuencia los textos del siglo XVII de François Poulain de la Barre De la igualdad de los dos sexos (1673) y La educación de las damas (1674), que formaban parte del debate a favor del acceso al saber por parte de las mujeres, aunque no existen datos sobre la difusión de estos libros en el círculo en el que se desenvolvía Mary Wollstonecraft. Tampoco está demostrado que tuviese conocimiento del valioso trabajo de Olympe de Gouges Los derechos de la mujer publicado en París un año antes que el propio texto de Wollstonecraft. Sin embargo, la escritora inglesa comparte la misma perspectiva intelectual de de Gouges y probablemente se benefició del denominado "polen de ideas" que se diseminaba por Europa desde finales del siglo XVII y que floreció en este caso en la última década del denominado siglo de las luces (Lorenzo 2004: 31).

Wollstonecraft polemiza abiertamente en su Vindicación de los derechos de la mujer con Jean- Jacques Rousseau. Este escritor y pedagogo francés ha pasado a la posteridad como defensor de los derechos civiles y de la educación. Sin embargo, en sus escritos, y particularmente en los de corte didáctico como Emilio; o, sobre la educación, publicado en 1762, deja claro que los derechos a los que él se refiere son solamente para "la mitad de la especie humana", en palabras de Wollstonecraft (317), porque segrega directamente a las mujeres en su propuesta educativa y dispone que sólo existan en función de los hombres:

[...] la educación de las mujeres siempre debe ser relativa a los hombres. Agradarnos, sernos de utilidad, hacernos amarlas y estimarlas, educarnos cuando somos jóvenes y cuidarnos de 
adultos, aconsejarnos, consolarnos, hacer nuestras vidas fáciles y agradables: éstas son las obligaciones de las mujeres durante todo el tiempo y lo que debe enseñárseles en la infancia. En la medida en que fracasamos en repetir este principio, nos alejamos del objetivo y todos los preceptos que se les da no contribuyen ni a su felicidad ni a la nuestra (Rousseau, Emilio, cit en Wollstonecraft 1996: 218).

En el año 1789, sentando las bases de la Revolución Francesa, la Asamblea Nacional redacta la denominada "Declaración de los derechos del hombre y de los ciudadanos", expresando así la voluntad popular de igualdad de derechos para todos los ciudadanos, en contraposición con la realidad vigente en el Antiguo Régimen. Tres años más tarde, en 1791, Olympe de Gouges publica "La Déclaration des Droits de la Femme et de la Citoyenne" ("Declaración de los derechos de la mujer y de la ciudadana"), texto paralelo al anterior en el que establece la necesidad de que se reconozcan exactamente los mismos derechos para las mujeres y las ciudadanas. De Gouges, tomando como referencia la declaración de la Asamblea Nacional en relación a los hombres, llega al punto de elaborar otros diecisiete artículos con el fin último y expreso de que esta declaración sea decretada también por la Asamblea Nacional. El texto de de Gouges, con una dedicatoria a la reina, presenta una introducción en la que apela directamente al género masculino y a su culpabilidad por la situación actual de las mujeres y la conculcación de sus derechos. Dice de Gouges:

Man $[\ldots]$, in this age of enlightenment and wisdom [...] wants to rule like a despot over a sex which has received all the intellectual faculties; he pretends to rejoice in the revolution, and to claim his rights to equality, in order to say no more about it (Gouges 2001: 380- 381).

Evidentemente Wollstonecraft, en su periplo francés debió estar al corriente de estas declaraciones de derechos y que Olympe de Gouges sería guillotinada dos años después. Lo que sí sabemos es que Mary Wollstonecraft no es ajena a la labor política llevada a cabo por Charles M. Talleyrand en el ejercicio de su cargo de diputado ni a su Informe sobre la instrucción pública, hecha en nombre del Comité de Constitución de la Asamblea Nacional los 10, 11 y 19 de septiembre de 1791. Wollstonecraft polemiza públicamente con este político y antiguo Obispo de Autun al dedicarle la Vindicación de los derechos de la mujer y rogarle por este medio que se evite la "coerción establecida en la sociedad" (Wollstonecraft 2001: 111) sobre las mujeres, como ella la denomina, y que esta discriminación no se perpetúe en la redacción de la nueva constitución francesa presentada en la Asamblea Constituyente.

El antedicho Informe sobre la instrucción pública de Talleyrand aboga por cinco principios de la educación, esto es, que debe ser accesible para todos, que -por endecualquier persona puede participar en la propagación de dicha educación; debe ser también universal, en el sentido de que llegue a todas las capas sociales, a hombres y a mujeres y para todas las edades. Esto se afirma con la rotundidad que se puede observar a continuación:

L'instruction doit exister pour l'un et l'autre sexe; cela est trop évident; puisqu'elle est un bien común, sus quel principe l'un des deux pourrait-il en étre déshérité par la societé protectrice des droits de tous? (Talleyrand 1992: 7).

No obstante lo anterior, en el desarrollo del punto específico relativo a la educación de las mujeres se las excluye del beneficio de esta educación, no 'de iure', pero sí 'de facto'. 
El análisis político que realiza es impecable en el sentido de que deben tener igualdad en la educación para aspirar a la igualdad social. La cuestión es que posteriormente apela a cuestiones como la felicidad social y la armonía de las parejas, y termina por concluir que la mujer desea continuar con su feliz vida doméstica y no aspira a tener protagonismo social ni político en aras de la felicidad y afirma lo siguiente

Le but de toutes les institutions doit être le bonheur du plus grand nombre. Tout ce qui s'en écarte est une erreur; tout ce qui y conduit, une vérité. Si l'exclusion des employs publics prononcée contre les femmes est pour les deux sexes un moyen d'augmenter la somme de leur bonheur mutual, c'est dès lors une loi que toutes les Sociétés ont dû reconnaitre et consacrer. Toute autre ambition serait un renversement des destinations premières; et les femmes n'auront jamais intérêt à changer la delegation qu'elles ont reçue.Or il nous semble incontestable que le bonheur commun, surtout celui des femmes, demande qu'elles n'aspirent point à l'exercice des droits et des fonctions politiques (Talleyrand 1992: 1).

Evidentemente, la reacción de Wollstonecraft es rápida y enérgica al plantear la desigualdad de trato social y legal existente y solicitar que esto sea modificado oficialmente, en contra de Talleyrand, que lo considera positivo:

Pero si se debe excluir a las mujeres, sin tener voz, de participar en los derechos naturales del género humano, pruebe primero, para rechazar la acusación de injusticia e inconsistencia, que carecen de razón; de otro modo, esta grieta en vuestra NUEVA CONSTITUCIÓN siempre mostrará que el hombre, de alguna forma, debe actuar como un tirano, y la tiranía, en cualquier parte de la sociedad donde alce su descarado frente, siempre socavará la moralidad. [...] Deseo, señor, sacar a flote algunas investigaciones de este tipo en Francia y si llevan a confirmar mis principios, cuando se revise vuestra constitución, debieran respetarse los Derechos de la Mujer, si se prueba plenamente que la razón exige este respeto y demanda en alta voz JUSTICIA para la mitad de la raza humana (Wollstonecraft 1996: 111- 112).

De este modo lo que hace Mary Wollstonecraft es tomar parte activa en la discusión constitucional francesa y plantear principios que, con el tiempo, serán asumidos por la civilización europea.

\section{LA TRADICIÓN EN EL REINO UNIDO}

Mary Wollstonecraft es una hija de su tiempo, en el sentido de que las pautas que informan su pensamiento provienen de los planteamientos de autores y autoras que la precedieron, algunos de manera consciente, ya que los cita reiteradamente en sus ensayos, y otros de manera indirecta. Sus posiciones pueden resumirse en varios principios básicos tal y como los expone en el capítulo de conclusiones de la Vindicación de los derechos de la mujer (Wollstonecraft 1996: 390- 394):

1. la libertad es la base de las virtudes cívicas,

2. la moral, tanto privada como pública debe basarse en principios inmutables,

3. los seres racionales deben guiarse únicamente por la autoridad de la razón,

4. debe producirse una Revolución en los modales femeninos basada en la formación y en la igualdad de derechos y deberes. 
Como es sabido, en 1688 en Inglaterra tuvo lugar la denominada Revolución Gloriosa, que surgió como rechazo al absolutismo del rey Carlos II de Inglaterra, y logró que el poder del parlamento limitase las prerrogativas de la monarquía, que sólo así fue aceptada de nuevo. Como resultado de la revolución, se declara la preeminencia de la confesión anglicana sobre las otras iglesias existentes en el Reino Unido, y se cambia a un rey católico -Carlos II- por una soberanía compartida por dos monarcas protestantes: Guillermo de Orange y María Estuardo. Una de las consecuencias más sobresalientes para la ciudadanía europea de esta 'revolución sin sangre' fue el avance irrefrenable del parlamentarismo sobre el poder de los monarcas, que hubieron de aceptar condiciones impuestas por la cámara para ser reconocidos como reyes.

Uno de los herederos intelectuales de esta revolución es Richard Price, un clérigo disidente y liberal que fue amigo y tutor académico de Wollstonecraft y que pertenecía al círculo de amistades que ella frecuentó en Londres. Las opiniones de Price pasaron a la posteridad gracias a la publicación del Discurso sobre el amor a nuestro país (1789) pronunciado en 1788 para conmemorar el centenario de la revolución inglesa en la Revolution Society. Quizás convendría aclarar que en inglés el término 'disidente' se refiere a un grupo de iglesias protestantes entre las que se encuentran los Presbiterianos, los Baptistas, los Quáqueros y otros grupos y que fueron perseguidos por no aceptar la supremacía de la iglesia anglicana. La legislación vigente les impedía asistir a las escuelas públicas o a la universidad, no podían reunirse en grupos y tenían que alejarse de sus iglesias y pedir autorización para todas sus actividades, etc. Luchaban por un futuro más justo e igualitario. Entre los disidentes más conocidos en España podemos citar, por ejemplo, a Daniel Defoe.

El círculo disidente frecuentado por Wollstonecraft influyó notablemente en su pensamiento. Ella elogió en particular la necesidad de reformas sociales en sintonía con lo expresado por Richard Price en su discurso conmemorativo de la revolución. Evidentemente, esta opinión no era unánime en el Reino Unido, ya que Price fue criticado ácidamente por Edmund Burke que lo tachó de ser demasiado progresista en su obra Reflexiones sobre la revolución en Francia (1790). Esto provoca que Wollstonecraft inicie una polémica con Burke en su Vindicación de los derechos de la mujer debido a lo poco ponderada crítica hecha al famoso pastor.

Otro de los antecedentes en las concepciones de la educación y de la organización cívica de la sociedad es el filósofo John Locke (1632-1704), al que Wollstonecraft cita en la Vindicación. Como se sabe, Locke se adelanta en cierta medida a Montesquieu defiende la separación de los poderes legislativo y judicial y la supremacía del primero sobre el segundo. También es conocida la defensa por parte del filósofo inglés de los derechos individuales y de la tolerancia, que suponen un claro antecedente del pensamiento democrático contemporáneo. Es con este marco intelectual y con las implicaciones en la educación y en otros campos con el que se alinea Mary Wollstonecraft (Lorenzo 2004: 26).

Las mujeres del período dieciochesco se ocupan en sus textos de forma reiterada de la necesidad de la educación para todas. No es posible hacer aquí relación de todos ellos. Las primeras discusiones sistematizadas sobre la mujer surgieron en Inglaterra a finales del siglo XVI, cuando se publicaron varios argumentos polémicos de mujeres en respuesta a afrentas masculinas. Estas críticas públicas eran el producto de la furia de los hombres ante 
la cada vez mayor presencia pública de mujeres, en un principio pertenecientes a la aristocracia o a familias adineradas. Estos escritos suponían una autoafirmación de las mujeres que se vio apoyada de algún modo por las ideas de igualdad religiosa propagadas por las sectas no-conformistas. Las mujeres pertenecientes a estas sectas radicales reivindicaron la libertad de palabra y acción y el derecho a desafiar a la Iglesia oficial y, por extensión, a la autoridad patriarcal. Como consecuencia, el número de mujeres que se dedicaron a la escritura fue en aumento, y cada vez más en serio, como una profesión. Así, tenemos el caso de Aphra Behn, que aunque no fue la primera mujer que se dedicó a escribir para ganarse el sustento, sí que fue la primera que lo hizo de forma profesional.

A finales del siglo XVII, Mary Astell (1668-1730) propugna la creación de centros de formación para las mujeres en su Propuesta seria a las mujeres. Para el avance de su interés mayor y verdadero de 1694, aunque lo hace de forma anónima.

El conocido novelista y disidente Daniel Defoe (1660-1731) publicó el artículo titulado "Una academia para mujeres", inserto en An Essay upon Projects en 1698, esto es, un año después de la publicación de la segunda parte de la obra de Astell. Como se sabe, Astell y Defoe no compartieron planteamientos ideológicos ni religiosos, ya que ella tenía una perspectiva sobre el gobierno del país claramente conservadora 'tory' y defendía a la Iglesia anglicana apasionadamente. Quizás estas posturas de Astell, aunque genuinas, fueron oportunas y contribuyeron a que su mensaje fuese más aceptable por el 'establishment' de su tiempo. No obstante, ambos tuvieron planteamientos similares en lo que se refiere a la formación de las mujeres. Así, en Un ensayo sobre proyectos Defoe expone públicamente su defensa de los derechos de las mujeres, especialmente a la educación en la línea que defendió, por ejemplo, Feijoo en España:

The Capacities of Women are supposed to be greater, and their Senses quicker than those of the Men; and what they might be capable of being bred to, is plain from some Instances of Female-Wit, which this Age is not without; which upbraids us with Injustice, and looks as if we denied Women the advantages of Education, for fear they should vie with the Men in their Improvements. / To remove this Objection, and that Women might have at least a needful Opportunity of Education in all sorts of Useful Learning, I propose the Draught of an Academy for that purpose (Defoe 1996: 492).

Sin embargo, durante el siglo XVIII esta opinión no era socialmente mayoritaria y las mujeres en su conjunto no ganaron terreno en sus esfuerzos por mantener su espacio y las funciones tradicionales desarrolladas en el mercado de trabajo debido a cambios sociales tales como el vallado de la tierra y la industrialización, y tampoco hubo avances significativos en la puesta en marcha den la educación reglada.

Esto ocasionó que muchas mujeres considerasen el matrimonio como medio de supervivencia económica. Wollstonecraft también coincide con autores precedentes en este punto puesto que cree vehementemente que el comportamiento de los seres humanos debe estar basado en la razón y en la rectitud de intenciones, y critica por tanto que a las mujeres se les inculque un modo de actuación dirigido al engaño de los hombres, en el mismo sentido que ya se había expresado Defoe en Conjugal Lewdness, or Matrimonial Whoredom. Lascivia Conyugal o prostitución matrimonial (1727), otro texto reformista que - con enfoque diferente - propugna un cambio de costumbres sociales. 
Otra de las pioneras en mostrar las ventajas de las academias para mujeres fue la novelista Sarah Fielding, que publicó en 1749 un libro titulado The Governess, or Little Female Academy. La Gobernanta o pequeña academia femenina, en la que se muestra la praxis de la educación, en el sentido Lockeano de inculcar valores de una educación en la solidaridad y regida por valores democráticos y no autoritarios y las ventajas que aporta para la vida de las mujeres (Lorenzo 1993). Cuando Mary Wollstonecraft funda su escuela femenina cerca de Londres sigue, pues, el pensamiento más vanguardista de los escritores y escritoras precedentes y trata de llevarlo a la práctica.

En esta revisión del contexto intelectual que precedió a la publicación de la Vindicación de los derechos de la mujer no podemos olvidar que la eclosión de la novela como género literario tuvo lugar en Inglaterra en el siglo XVIII. Buena parte de este fenómeno se debe a las mujeres, que fueron quienes mayoritariamente contribuyeron a ello tanto como autoras como, especialmente en su calidad de lectoras. Aunque los textos de ficción tuvieron muy diversas funciones en el siglo XVIII, su uso como forma no reglada de instrucción de las mujeres es hoy comúnmente aceptado. La propia Wollstonecraft, que criticó en la Vindicación de los derechos de la mujer la novela sentimental por sus características alienantes (Wollstonecraft 1996: 378) publicó textos de ficción tales como María, un relato (1788), antes del texto ensayístico por el cual ha pasado a la posteridad. La base de su pensamiento en relación con el género novelesco, y por extensión de las novelas sentimentales, es que conviene fomentar la educación mediante la razón, y no alienarlas mediante las emociones o la evasión que la lectura de ficción podía proporcionar, perpetuando así el 'statu quo'.

Entre las novelistas que dieron a la imprenta textos en los que se propugnaba la mejora en la instrucción femenina y en su comportamiento social según pautas morales no mercantilistas se encuentran Mary Davys, Eliza Haywood, Frances Sheridan y la propia Jane Austen. Mary Davys (1674- 1732) publicó en el año 1725 Familiar Letters, novela epistolar en la que la protagonista pretende regir su comportamiento de acuerdo con sus pautas religiosas y políticas, mucho más modernas que su 'partenaire' masculino. Eliza Haywood (1693?- 1756) es una figura sobresaliente en cuanto a la pujanza de sus heroínas y sus capacidades para tomar las riendas de sus propios destinos abandonando comportamientos superficiales, como sucede en Miss Betsy Thoughtless (1751). Haywood plantea así en la ficción perfiles de mujeres que estarían en sintonía con los planteamientos de Wollstonecraft. Frances Sheridan (1724- 1766) en Memoirs of Miss Sidney Bidulph (1761) relata la historia de una joven que no desea casarse y expresa sus deseos de vida independiente evitando así matrimonios mercenarios en aras de su integridad personal y moral, planteamientos éstos que Wollstonecraft hace suyos como base para una sociedad tengan los mismos derechos que los hombres.

Una de las escritoras contemporáneas con las que se alinea Wollstonecraft en cuanto que comparte sus postulados relativos a la universalidad de la educación es Catherine Macaulay. En su ensayo Letters on Education (1790) (Cartas sobre la educación) Macaulay ya había anticipado también la crítica a Rousseau y a su consideración de que las mujeres no tenían los mismos derechos de los hombres. Macaulay representa, pues, uno de los apoyos intelectuales reconocidos por Wollstonecraft en su Vindicación de los derechos de la mujer. 
Podemos concluir que Wollstonecraft cataliza el pensamiento liberal precedente y lo hace visible, probablemente atacando la misoginia de forma más radical que otras voces, pero no representa una absoluta novedad en el panorama intelectual europeo del siglo XVIII. Su obra tiene todavía una sorprendente vigencia en el siglo presente, en su doble vertiente de pionera que marcó puntos de partida y de autora de vanguardia que apunta a objetivos todavía lejanos en este momento.

\section{REFERENCIAS BIBLIOGRÁFICAS}

ASTELl, M., A Serious Proposal To the Ladies, For the Advancement of their true and greatest Interest. By a Lover of Her Sex, Londres, R. Wilkin at the King's Head in St. Paul's Church-Yard, 1694. A Serious Proposal to the Ladies. Part II. Wherein a Method is offer'd for the improvement of their minds, London, R. Wilkin, 1697. A Serious Proposal to the Ladies: Parts I and II, ed. e introd. Patricia Springborg, Toronto, Broadview, 2002.

BURKE, E. Reflections on the Revolution in France, 1790, ed. e Introd. L.G. Mitchell, Oxford, Oxford University Press, 1993. Reflexiones sobre la revolución en Francia, ed. Carlos Mellizo, Madrid, Alianza, 2003.

DAVYS, M., Familiar Letters Betwixt a Gentleman and a Lady. Reimpresión facsimile en The Reform'd Coquet and Familiar Letters Betwixt a Gentleman and a Lady by Mary Davys. The Mercenary Lover by Eliza Haywood, Nueva York, Garland, 1973, pp. 264- 307.

DEFOE, D., “An Academy for Women,” An Essay upon Projects, 1698. Rpt. Robert DeMaria Jr ed. British Literature 1640-1789. An Anthology, Oxford, Blackwell, 1996, pp. $491-497$.

DEFOE, D., Conjugal Lewdness, or Matrimonial Whoredom, Nueva York, Scholar's Facsimiles \& Reprints, 1967.

FEIJÓO, B.J., Defensa de la mujer. Discurso XVI del Teatro crítico, Barcelona, Icaria, 1997.

GOUGES, O de., The Rights of Woman, en The Vindications. The Rights of Men. The Rights of Woman, 1997, ed. D. L. Macdonald \& Kathleen Scherf, Canada, Broadview Press, 2001, pp. 378- 392.

HAYWOOD, E., The History of Miss Betsy Thoughtless, London, Pandora, 1986.

KITTS, S.A., "Mary Wollstonecraft's A Vindication of the Rights of Woman: A Judicious Response from Eighteenth-century Spain", The Modern Language Review, 89.2 1994, pp. 351- 359.

LORENZO MODIA, M. J., Introducción. Vindicación dos dereitos da muller de Mary Wollstonecraft, Santiago de Compostela, Sotelo Blanco, 2004, pp. 13- 50.

LORENZO MODIA, M. J., "Literatura femenina didáctica: The Governess de Sarah Fielding." Simposio "Didáctica de lenguas y culturas," ed. Alfredo Rodríguez López-Vázquez, A Coruña, Universidade a Coruña, 1993, pp. 221- 225.

MACAULAY GRAHAM, C., Letters on Education.With Observations on Religious and Metaphysical Subjects, London, C.Dilly, 1970 (Reimp. Nueva Cork, Garland, 1974) 
PAJARES, E., "Inés Joyes y Blake, feminista ilustrada del XVIII." Boletín de la Biblioteca Menéndez Pelayo, LXXVI, 2000, pp. 181-192.

PALACIOS FERNÁNDEZ, E., La mujer y las letras en la España del siglo XVIII, Madrid, Ediciones del Laberinto, 2002.

POULAIN DE LA BARRE, F., De la educación de las damas: para la formación del espíritu en las ciencias y en las costumbres, ed. y trad. Ana Amorós, Madrid, Cátedra, Universitat de València, Instituto de la Mujer, 1993.

POULAIN DE LA BARRE, F., The Equality of the Sexes [1673], ed. y trad. Desmond M. Clarke, Manchester, Manchester UP, 1990.

PRICE, R., A Discourse on the Love of our Country, Delivered on Nov. 4, 1789, at the Meeting-House in the Old Jewry, to the Society for Commemorating the Revolution in Great Britain. With an Appendix, Containing the Report of the Committee of the Society; an Account of the Population of France; and the Declaration of Rights by the National Assembly of France, London, T. Cadell, 1789 (Oxford, Woodstock, 1992).

ROUSSEAU, J.J., Emile, ou De L'éducation, Paris, Garnier- Flammation cop., 1966. Emilio, o de la educación, Madrid, EDAF, 1995.

SAU, V. Introducción. Defensa de la mujer. Discurso XVI del Teatro crítico de Benito J. Feijóo, Barcelona, Icaria, 1997, pp. 9- 11.

SHERIDAN, F. Memoirs of Miss Sydney Bidulph. Extracted from her own Journal, and now first published. In Three Volumes, Londres, Dodsley, 1761 (Oxford, Oxford UP, 1995).

TALLEYRAND P., CHARLES M. Rapport sur l'instruction publique, fait au nom du Comité de Constitution à l'Assemblée nationale, les 10, 11 et 19 setembro 1791 [Document électronique], París, Maxwell, 1992.

WOLLSTONECRAFT, M., A Vindication of the Rights of Men, in a Letter to the Right Honourable Edmund Burke. Occasioned by his Reflections on the Revolution in France, London, Joseph Johnson, 1790, en The Vindications. The Rights of Men. The Rights of Woman, 1997, ed. D. L. Macdonald \& Kathleen Scherf, Canada, Broadview Press, 2001, pp. 31- 98.

WOLLSTONECRAFT, M., A Vindication of the Rights of Woman, With Strictures on Political and Moral Subjects, London: Joseph Johnson, 1792, ed. D. L. Macdonald \& Kathleen Scherf; Canada, Broadview Press, 2001, pp. 99- 343. Vindicación de los Derechos de la Mujer; Madrid, Debate, 1977. Feminismos, ed. Isabel Burdier, trad. Carmen Martínez Gimeno, Universitat de València: Cátedra; Instituto de la Mujer, Madrid (1994, 1996).

WOlLStOnECRAFT, M., Mary, A Fiction, London: Joseph Johnson [1788]. Mary and The Wrongs of Woman, ed. Gary Kelly, Oxford, Oxford University Press, 1998, pp. 1- 70 . 